\title{
Book Review: Tried, Revised, and Tried Again-A Seasoned Textbook for Undergraduates
}

\author{
Cell and Molecular Biology: Concepts and Experiments, 3rd ed., by Gerald Karp; 854 pp.; \\ Wiley (New York)
}

Reviewed by Christopher Watters, Middlebury College

Submitted June 24, 2002; Revised June 29, 2002; Accepted July 1, 2002

In 1999 I adopted the second edition of Karp's text (CMB2) for my introductory lecture-and-laboratory course in cellular and molecular biology, and this past spring I began using the newly revised third edition (CMB3). Clearly, I like the text, and I had helped the author revise two chapters (4 and 8) for the current edition. I also surveyed how my students perceived both editions, and what follows are our impressions of the third edition, with echoes of sentiment expressed about the second edition.

First, let me provide some background information about how I use CMB at Middlebury College, in what is the final course in a three-unit biology core, which also includes laboratory courses in ecology and in genetics and evolution. Students using this text are mainly sophomores who have had one introductory laboratory course in genetics and evolution and at least one laboratory chemistry course. Many of the students are simultaneously enrolled in an organic chemistry course, and about two-thirds of the class are declared preprofessional students (preparing for medical, dental, or veterinary programs); about a quarter of these are nonscience majors. Each week, we meet for $3 \mathrm{~h}$ of lecture and discussion, a 2-h evening session devoted to problem solving and prelab instruction or for periodic examinations, and $3 \mathrm{~h}$ of laboratory work. Students and I approach cellular and molecular biology analytically and discursively, in both an intensive manner and an extensive manner, and I tend to use a text primarily as an up-to-date source of accurate information and jargon. I inevitably sacrifice coverage in favor of discussion and analysis, and we cover what we can during the College's 12-week term (usually about two-thirds of the chapters). I have found $\mathrm{CMB}$ eminently suitable for these students and this pedagogy. However, it would not work well as a first-year text or for a general audience wanting to satisfy a science requirement. Similarly, upper-level undergraduates and graduate students requiring more depth and detail would be better served by either of the longer, recently revised texts by Alberts et al. (2002) or Lodish et al. (1999).

I chose CMB for the quality of both its prose and its illustrations, for its currency and level of detail, and for its generally analytic approach to its topics. Karp's writing is clear,

DOI: $10.1187 /$ cbe.02-07-0019

Corresponding reviewer. E-mail address: watters@middlebury. edu. usually crisp, and in context appropriately discursive and often synthetic as well as analytical. His coverage is usually accurate, and although often dense with detail, his prose is well seasoned with bold and italic type to emphasize important points and concepts. Each chapter begins with a generally well-focused overview; is well punctuated with brief, often provocative review questions; and ends with an excellent synopsis, a bibliography, and review questions and problems graduated in difficulty. Moreover, chapters are interspersed with extended asides that provide interesting detail concerning clinical perspectives or experimental pathways and that greatly leaven even the denser material. Many students complain, at least initially, about the complexity of the material, the amount of detail, and the vocabulary. However, they find the extensive glossary helpful but make only occasional use of the index. Whether they master the introductory aspects of cellular and molecular biology (or, more specifically, my essay and problem-oriented examinations) depends, I think, on the degree to which they successfully grapple with the prose and the jargon, and most agree, apparent detail and density aside, that the text is clearly written.

From my students' perspective, the illustrations are the text's most striking and educationally beneficial feature, in both the second and the third editions. The figures are expertly drawn (or in the case of photographs, well reproduced), strikingly colored, and appropriately detailed; and there are lots of them. The key figures in various chapters have enlarged inset views of important details, which renders them somewhat "telescoped" in appearance; this feature usually improves their depth of coverage without sacrificing their clarity. Although only some of the figures are provided in the instructor's kit as transparencies, all are available on the instructor's CD-ROM as PowerPoint slides. Many chapters include illustrations of macromolecular structures that have been created by RasMol or comparable software programs, and the author might consider including a description of these modeling tools in the Methods chapter of the fourth edition. Several chapters in CMB3 (notably those on membrane structure and function and on the cytoskeleton and cell motility) have been considerably enhanced by the significant addition of new figures. In sum, I consider many figures comparable in quality to those found in the longer, more comprehensive texts (Alberts et al., 2002; Lodish et al., 1999), and CMB4 and future editions would benefit from the addition of animated 
gifs and movies similar to what is available the on CDs accompanying the longer texts.

Karp's illustrations are an integral part of CMB and critical to its success as an educational resource in my course. In fact, many students report that they approach the text, and review their lecture notes, primarily through the figures, in both the text and the accompanying $C D$, coming to the prose secondarily (or later in their study). It is fortunate in this regard that Karp describes most figures in detailed, clearly written, and well-referenced captions. Although the captions are usually, and usefully, redundant of the more detailed and comprehensive text coverage, students and I have been mildly irritated by occasional figure detail mentioned in a caption but not described elsewhere in the accompanying text. It is also critical that, given their perceived importance, key figures not be so telescoped as to confound already complex detail. For the most part, Karp's figures are excellent in this regard and for the clarity of their detail, but there are occasional lapses. For example, the figure (8.29) describing the targeting of lysosomal enzymes illustrates in part (b) the movement through the endomembrane system of a luminal enzyme tagged by phosphorylated mannose and its receptor $(\mathrm{MPR}$, mannose-6-phosphate receptor-an integral membrane protein, or IMP). However, part (a) of this figure illustrates the steps in the phosphorylation of the mannose residues of an IMP (not a luminal enzyme). Both parts of the figure are accurate, and reflect text descriptions, but at first (and often second) glance, naïve students are rightly confused about whether the MPR likewise has phosphorylated mannose residues or whether a lysosomal IMP also requires an MPR for correct targeting. Such issues are, perhaps rightly, not explored in an introductory text, but unnecessary confusion could have been avoided by illustrating the phosphorylation of a luminal enzyme in part (a) in parallel with its targeting pathway depicted in part (b).

CMB2 was the first of its kind (viz, shorter texts in cellular and molecular biology) to integrate discussion of the cytoskeletal structure and function and cell motility in a single chapter. Its chapter on cell signaling also provided unusually extensive, well-integrated, and current coverage of that subject. Such strengths are maintained in the current edition, and new material has been introduced to many chapters to update topics and rectify deficiencies in the second edition (including, for example, ion channel structure and function, actin polymerization and cellular motility, and the use of green fluorescent protein and DNA microarrays). Moreover, many chapters are now replete with illustrations of molecular structures in concert with the discussion of their functions. However, unless carefully done, the addition of new information can disrupt what was originally well-integrated coverage, and organizational lesions are beginning to appear in the chapters just mentioned. In addition, some chapters seem little changed: for example, except for an excellent overview figure and discussion of the phenomenon of photoinhibition, the coverage of photosynthesis appears as perfunctory as it did in the second edition (perhaps reflecting similar, only nominal coverage in many introductory courses in cellular and molecular biology). In contrasts, the index of CMB3 has been expanded and more thoroughly cross-referenced.

Possibly, the major shortfall of what is otherwise a generally excellent revision of a very good text is a niggling lack of thoroughness about details, both old and new. (The text editor(s) and outside reviewers, such as me, bear some responsibility for oversights, which unfortunately often become evident only in the give-and-take of a good class.) Several examples may help illustrate this point. It is now clear that guanosine triphosphate (GTP)-binding proteins (aka G proteins or slow GTP hydrolases) regulate a variety of cellular functions in ways that reflect the conformational changes that accompany their enzymatic cycle. However, Karp names the various forms inconsistently and rarely when talking about one form does he refer the reader to other examples. Moreover, any systematic treatment of their operation as signal mediators is restricted to discussion of the heterotrimeric form and of Ras. Perhaps as a consequence, and this is my point, the descriptions of the numerous kinds of $\mathrm{G}$ proteins are uneven in detail and perspective, and an invaluable opportunity for conceptual synthesis is lost. Thus, when the function of Rab proteins is described as initiating vesicular tethering, the accompanying figure illustrates the Rab protein in its GTP-bound form, but no mention is made of the guanosine diphosphate (GDP) form, what happens when hydrolysis converts the former to the latter, or whether guanine nucleotide exchange factors (GEFs) or GTPase-activating proteins (GAPs) are or might be involved in Rab regulation. Occasionally, also, revision has added erroneous information: for example, caffeine does not increase the likelihood that ryanodine-sensitive $\mathrm{Ca}^{2+}$ channels will remain open by lowering the channels' sensitivity to calcium, as indicated in the text, but by increasing their sensitivity. Good classroom discussion can be generated from such lapses and editorial oversights, and although no single author can cover all details and all perspectives, problems of this sort, unless corrected, tend to propagate through successive editions, which generates more frustration than enlightenment among even the most serious student.

In summary, CMB3 is an able successor to its predecessor and so far Karp seems firmly on track with his goal of revising the text every 3 yr. He provides a consistency of style and perspective often lacking in multiauthored texts, but it will be interesting to see whether with each arithmetic edition he can also continue the extensive coverage of the various disciplines and revise his chapters in a systematic and integrated manner as cellular and molecular biology continue to expand in their geometric fashion.

\section{REFERENCES}

Alberts, B., Johnson, A., Lewis, J., Raff, M., Roberts, K., and Walter, P. (2002). Molecular Biology of the Cell, 4th ed., New York: Garland.

Lodish, H., Berk, A., Zipursky, L., Matsudaira, P., Baltimore, D., and Darnell, J.E. (1999). Molecular Cell Biology, 4th ed., New York: Freeman. 